\title{
Factors influencing to the implementation success of big data analytics: a systematic literature review
}

\begin{abstract}
Big data analytics (BDA) readiness factors have been widely researched; nevertheless, few have investigated the impact and success factors of BDA implementation in the organizational context. The relevance and quality of BDA outcomes have been a significant concern to the organizational leaders in supporting them for strategic decision-making. To that end, the objective of this study is twofold. Firstly, it investigates the factors that influencing the success of BDA implementation for effective decision-making. Secondly, this study adds to the body of knowledge in the information system (IS) domain, especially with its focus on BDA implementation packages. Based on 18 selected papers, this review has established 10 influencing factors that may influence the success of BDA implementation, therefore, contribute to the practice and research of BDA domain and its effectiveness towards the organizational performance enhancement.
\end{abstract}

Keyword: Big data analytics; Capabilities; Quality; Big data analytics implementation 